\title{
PONTRYAGIN'S MAXIMUM PRINCIPLE FOR OPTIMAL CONTROL OF A NON-WELL-POSED PARABOLIC DIFFERENTIAL EQUATION INVOLVING A STATE CONSTRAINT
}

\author{
MI JIN LEE' ${ }^{\prime}$ and JONG YEOUL PARK ${ }^{1}$
}

(Received 25 August, 2003; revised 18 November, 2003)

\begin{abstract}
In this paper, we study Pontryagin's maximum principle for some optimal control problems governed by a non-well-posed parabolic differential equation. A new penalty functional is applied to derive Pontryagin's maximum principle and an application for this system is given.
\end{abstract}

\section{Introduction}

In this paper, we shall study Pontryagin's maximum principle for optimal control of a non-well-posed parabolic differential equation of the form

$$
\begin{array}{ll}
\frac{\partial y}{\partial t}+A y+f(x, t, y)=u \quad \text { in } & Q=\Omega \times(0, T), \\
y=0 \quad \text { on } \quad \Sigma=\partial \Omega \times(0, T) & y(0)=y_{0},
\end{array}
$$

with the state constraint $F(y) \subset S$, where $\Omega$ is a bounded open subset with smooth boundary $\partial \Omega, f$ is a continuous function, $F: L^{2}(Q) \rightarrow X$ and $X$ is a Banach space. The cost functional is given by

$$
J(y, u)=\int_{0}^{T}[g(t, y(t))+h(u(t))] d t .
$$

Solving the control problem for infinite-dimensional systems and observations has been a big issue in the area of mathematical systems theory for more than thirty years. In the memorial work of Lions [5], linear optimal control theory for distributed

\footnotetext{
'Department of Mathematics, Pusan National University, Pusan 609-735, Korea; e-mail: jin8719@hanmail.net and jyepark@pusan.ac.kr.

(C) Australian Mathematical Society 2004, Serial-fee code 1446-1811/04
} 
parameter systems was developed to a considerable extent by using fundamental results on the existence and regularity of solutions to linear partial differential equations. Barbu $[1,2]$ contributed greatly to optimal control theory for nonlinear systems. The theory of nonlinear accretive operators and of nonlinear differential equations of accretive type has occupied an important place among functional methods in the theory of nonlinear partial differential equations since its inception in the 1960s. Its areas of application include existence theory for nonlinear elliptic and parabolic boundary value problems and problems with a free boundary.

For another approach to the optimal control problem using the maximal principle for optimal control for some nonlinear equations, we refer to $\mathrm{Li}$ and Yong [4] for example. Casas et al. [3] studied Pontryagin's maximum principle for some parabolic equation with gradient state constraints. Raymond and Zidani [7] considered time-optimal control problems governed by semilinear parabolic equations with pointwise state constraints and unbounded controls and derived a Pontryagin's principle for boundary controls. In particular, in recent years, there has been growing interest in Pontryagin's principles for control problems governed by non-well-posed differential equations. Some systems may have no global solution (global in time), or have more than one solution for each control. The optimal control problems governed by such systems are called non-well-posed optimal control problems. Wang $[8,10]$ dealt with optimal control problems for a non-well-posed elliptic differential equation. Moreover, Wang [9] obtained Pontryagin's maximum principle for parabolic differential equations with two-point boundary state constraints.

The purpose of this paper is to derive Pontryagin's maximum principle for optimal control of a non-well-posed parabolic differential equation involving state control. In particular, this paper deals with the cost functional which may be non-smooth and a convex set of controls while Wang [9] dealt with a non-convex control set. To derive Pontryagin's maximal principle, we shall introduce a new penalty functional which can transform the original optimal control problem into an optimisation problem with a parameter and use the method in Barbu $[1,2]$ to obtain the necessary conditions for the optimal solution of the optimisation problem.

The paper is organised as follows. In Section 2, we present the preliminaries and hypotheses of the paper. In Section 3, we derive Pontryagin's maximum principle for optimal control of a non-well-posed parabolic differential equation. Finally, in Section 4 , we give an application.

\section{Preliminaries}

Throughout this paper, we denote by $\Omega \subset R^{n}, n \geq 3$, a bounded open subset with smooth boundary $\partial \Omega$. Let $Q=\Omega \times(0, T)$ for some fixed $T>0$ and $\Sigma=\partial \Omega \times(0, T)$. 
We denote by $(,)_{\Omega}$ the inner product in $L^{2}(\Omega)$ and by $(,)_{Q}$ the inner product in $L^{2}(Q)$. Let $Y=H^{2,1}(\Omega) \cap L^{2}\left(0, T: H_{0}^{1}(\Omega)\right)$ where

$$
H^{2.1}(Q)=\left\{y \in L^{2}(Q): \frac{\partial y}{\partial t}, \frac{\partial y}{\partial x_{i}}, \frac{\partial^{2} y}{\partial x_{i} \partial x_{j}} \in L^{2}(Q) ; i, j=1, \ldots, n\right\}
$$

and

$$
\begin{aligned}
& W_{0}^{1,2 n /(n+2)}(\Omega)=\left\{w \in L^{2 n /(n+2)}(\Omega): \frac{\partial w}{\partial x_{i}} \in L^{2 n /(n+2)}(\Omega), i=1, \ldots, n,\right. \\
& w=0 \text { on } \partial \Omega\} \text {. }
\end{aligned}
$$

Let $(K, d)$ be a separable metric space and define $U=\{u: Q \rightarrow K$ is measurable $\}$ and $\tilde{d}(u, v)=\tau(\{(x, t) \in Q: u(x, t) \neq v(x, t)\})$, where $\tau$ denotes the Lebesgue measure in $R^{n}$. Then $(U, d)$ is a complete metric space.

The problem $(P)$ studied in this paper is as follows:

$$
\text { determine inf } J(y, u)=\inf \int_{0}^{T}[g(t, y(t))+h(u(t))] d t
$$

such that

$$
\begin{aligned}
& \frac{\partial y(x, t)}{\partial t}+A y(x, t)+f(x, t, y(x, t))=u(x, t) \text { in } Q, \\
& y(x, t)=0 \text { on } \Sigma, \quad y(x, 0)=y_{0},
\end{aligned}
$$

with the state constraint $F(y) \subset S$.

We assume the following hypotheses.

$\left(H_{1}\right)$ Let $A$ be the second-order elliptic differential operator

$$
A y=-\sum_{i, j=1}^{n} \frac{\partial}{\partial x_{i}}\left(a_{i, j}(x) \frac{\partial y}{\partial x_{j}}\right)
$$

where $a_{i, j} \in C^{1}(\bar{\Omega}), a_{i, j}(x)=a_{j, i}(x)$ in $\Omega$ for all $i, j=1, \ldots, n$ and for some $c_{0}>0$,

$$
\sum_{i, j=1}^{n} a_{i, j}(x) \theta_{i} \theta_{j} \geq c_{0}\left(\theta_{1}^{2}+\cdots+\theta_{n}^{2}\right)
$$

for all $x \in \bar{\Omega}$ and $\left(\theta_{1}, \ldots, \theta_{n}\right)$ in $R^{n}$.

$\left(H_{2}\right) \quad g:[0, T] \times L^{2}(\Omega) \rightarrow R^{+}$is measurable in $t$ and for every $r>0$ there exists $L_{r}>0$ independent of $t$ such that $g(t, 0) \in L^{\infty}(0, T)$ and

$$
|g(t, y)-g(t, z)| \leq L_{r}\|y-z\|_{H}
$$

for all $t \in[0, T],\|y\|_{H}+\|z\|_{H} \leq r$. 
$\left(H_{3}\right)$ The functional $h: U \rightarrow \bar{R}$ is convex and lower semicontinuous (l.s.c.). Moreover, there exist $c_{1}>0$ and $c_{2} \in R$ such that $h(u) \geq c_{1}\|u\|_{U}^{2}+c_{2}$ for all $u \in U$. $\left(H_{4}\right) f: \bar{\Omega} \times[0, T] \times R \times K \rightarrow R$ is continuous and $f_{y}^{\prime}: \bar{\Omega} \times[0, T] \times R \times K \rightarrow R$ is continuous, where $f_{y}^{\prime}$ denotes the derivative of $f$ to the third variable. Moreover,

$$
|f(x, t, y)| \leq \Lambda_{1}\left[a_{1}(x, t)+|y|^{r_{1}}\right]
$$

for all $(x, t) \in \bar{Q}, y \in R$ and $u \in K$, where $\Lambda_{1} \geq 0, a_{1} \in L^{2}(Q), a_{1}(x, t) \geq 0$ a.e. in $Q, 1 \leq r_{1} \leq n /(n-2)$. Also

$$
\left|f_{y}^{\prime}(x, t, y)\right| \leq \Lambda_{2}\left[a_{2}(x, t)+|y|^{r_{1}-1}\right]
$$

for all $(x, t) \in \bar{Q}, y \in R$ and $u \in K$, where $\Lambda_{2} \geq 0, a_{2} \in L^{n}(Q), a_{2}(x, t) \geq 0$ a.e. in $Q$.

$\left(H_{5}\right)$ Let $X$ be a Banach space with the dual $X^{*}$ strictly convex. Let $S \subset X$ be a closed convex subset with finite codimensionality. Then $F: L^{2}(Q) \rightarrow X$ is of class $C^{1}$.

Let $\left(y^{*}, u^{*}\right)$ be optimal for problem $(P)$. In order to get the maximum principle for $\left(y^{*}, u^{*}\right)$, we need the following additional assumption.

$\left(H_{6}\right) \quad F^{\prime}\left(y^{*}\right) D_{r}-S$ has finite codimensionality in $X$ for some $r>0$, where $D_{r}=$ $\left\{z \in Y:\|z\|_{Y} \leq r\right\}$.

\section{Pontryagin's maximum principle for $(P)$}

In order to introduce the approximating control process, we give approximations $g^{\varepsilon}$ of $g$ and $h_{\varepsilon}$ of $h$ as follows. The approximation $g^{\varepsilon}:[0, T] \times L^{2}(\Omega) \rightarrow R$ is defined by

$$
g^{\varepsilon}(t, y)=\int_{R^{N}} g\left(t, p_{N} y-\varepsilon \Lambda_{N} \tau\right) \rho_{N}(\tau) d \tau,
$$

where $\rho_{N}$ is a mollifier in $R^{N}, N=\left[\varepsilon^{-1}\right], p_{N}: L^{2}(\Omega) \rightarrow X_{N}$ is the projection $L^{2}(\Omega)$ on $X_{N}$ which is the finite-dimensional space generated by $\left\{e_{i}\right\}_{i=1}^{N}$, where $\left\{e_{i}\right\}_{i=1}^{\infty}$ is an orthonormal basis in $L^{2}(\Omega)$. Here $\Lambda_{N}: R^{N} \rightarrow X_{N}$ is the operator defined by $\Lambda_{N}(\tau)=\sum_{i=1}^{N} \tau_{i} e_{i}, \tau=\left(\tau, \tau_{2}, \ldots, \tau_{N}\right)$. The approximation $h_{\varepsilon}: L^{2}(\Omega) \rightarrow \bar{R}$ is defined by

$$
h_{\varepsilon}(u)=\inf \left\{\|u-v\|_{L^{2}(\Omega)}^{2} /(2 \varepsilon)+h(u): u \in L^{2}(\Omega)\right\} .
$$

Now we introduce the following approximation problem $\left(P^{\varepsilon}\right)$ :

$$
\text { determine inf } J_{\varepsilon}(y, u) \text { over all }(y, u) \in Y \times L^{2}(\Omega),
$$


where

$$
\begin{aligned}
J_{\varepsilon}(y, u)= & \int_{0}^{T} g^{\varepsilon}(y) d t+\int_{0}^{T} h_{\varepsilon}(u) d t+\frac{1}{2}\left\|u-u^{*}\right\|_{L^{2}(0, T: U)}^{2} \\
& +\frac{1}{2 \tilde{r}} \int_{Q}\left|y-y^{*}\right|^{2 \tilde{r}} d x d t+\frac{1}{2 \varepsilon}\left[\varepsilon+d_{S}(F(y))\right]^{2} \\
& +\frac{1}{2 \varepsilon}\left\|\frac{\partial y}{\partial t}+A y+f(x, t, y)\right\|_{L^{2}(Q)}^{2} .
\end{aligned}
$$

Here $\tilde{r}=n /(n-2)$. Note that by Sobolev's imbedding theorem, $Y \subset L^{2 \bar{r}}(\Omega)$. Thus for each $\varepsilon>0, J_{\varepsilon}$ is well defined on $Y \times L^{2}(\Omega)$.

LEMMA 3.1. Problem $\left(P^{\varepsilon}\right)$ has at least one solution.

PROOF. Let $d=\inf _{(y, u) \in Y \times L^{2}(Q)} J_{\varepsilon}(y, u)$. By the hypotheses $\left(H_{2}\right)$ and $\left(H_{3}\right)$ it is obvious that $d>-\infty$. Let $\left(y_{m}, u_{m}\right) \in Y \times L^{2}(Q)$ be such that

$$
d \leq J_{\varepsilon}\left(y_{m}, u_{m}\right) \leq 1 / m+d .
$$

By virtue of (3.1), $\left\{\left(y_{m}, u_{m}\right)\right\}$ is bounded in $L^{2 \tilde{r}}(Q) \times L^{2}(Q)$ and $\{(\partial y / \partial t)+A y+$ $f(x, t, y)\}$ is bounded in $L^{2}(Q)$. By $\left(H_{4}\right),\{f(x, t, y)\}$ is bounded in $L^{2}(Q)$. Thus $\left\{y_{m}\right\}$ is bounded in $Y$. Therefore we can extract a subsequence, still denoted by $\left\{y_{m}, u_{m}\right\}$, such that

$$
\begin{aligned}
& u_{m} \rightarrow \tilde{u} \text { weakly in } L^{2}(Q) \text { as } m \rightarrow \infty, \\
& y_{m} \rightarrow \tilde{y} \text { weakly in } Y \text { as } m \rightarrow \infty \\
& y_{m}(x) \rightarrow \tilde{y}(x) \text { a.e. in } Q \text { as } m \rightarrow \infty
\end{aligned}
$$

On the other hand, since $f$ is continuous, we have that

$$
f\left(x, t, y_{m}(x)\right) \rightarrow f(x, t, \tilde{y}(x)) \text { a.e. in } Q \text { as } m \rightarrow \infty \text {. }
$$

Since $\left\{f\left(x, t, y_{m}\right)\right\}$ is bounded in $L^{2}(Q)$, by (3.4), we get that

$$
f\left(x, t, y_{m}\right) \rightarrow f(x, t, \tilde{y}) \text { weakly in } L^{2}(Q) \text { as } m \rightarrow \infty .
$$

Hence

$$
\frac{\partial y_{m}}{\partial t}+A y_{m}+f\left(x, t, y_{m}\right) \rightarrow \frac{\partial \tilde{y}}{\partial t}+A \tilde{y}+f(x, t, \tilde{y}) \text { weakly in } L^{2}(Q) .
$$

By (3.5), it follows that

$$
\liminf _{m \rightarrow \infty}\left\|\frac{\partial y_{m}}{\partial t}+A y_{m}+f\left(x, t, y_{m}\right)\right\|_{L^{2}(Q)}^{2} \geq\left\|\frac{\partial \tilde{y}}{\partial t}+A \tilde{y}+f(x, t, \tilde{y})\right\|_{L^{2}(Q)}^{2} .
$$


By (3.3) and by Sobolev's imbedding theorem,

$$
y_{m} \rightarrow \tilde{y} \quad \text { strongly in } L^{2}(Q) \text { as } m \rightarrow \infty
$$

By (3.7) and by hypothesis $\left(H_{6}\right)$, we have that

$$
\frac{1}{2 \varepsilon}\left[\varepsilon+d_{S}\left(F\left(y_{m}\right)\right)\right]^{2} \rightarrow \frac{1}{2 \varepsilon}\left[\varepsilon+d_{S}(F(\tilde{y}))\right]^{2} \quad \text { as } m \rightarrow \infty .
$$

On the other hand, by (3.3) and by the hypotheses $\left(\mathrm{H}_{2}\right)$ and $\left(\mathrm{H}_{3}\right)$, we deduce that

$$
\begin{gathered}
\liminf _{m \rightarrow \infty}\left\{\int_{0}^{T}\left(g^{\varepsilon}\left(y_{m}\right)+h_{\varepsilon}\left(u_{m}\right)\right) d t+\frac{1}{2} \int_{Q}\left(u_{m}-u^{*}\right)^{2} d x d t+\frac{1}{2 \tilde{r}} \int_{Q}\left|y_{m}-y^{*}\right|^{2 \tilde{r}} d x d t\right\} \\
\geq \int_{0}^{T}\left(g^{\varepsilon}(\tilde{y})+h_{\varepsilon}(\tilde{u})\right) d t+\frac{1}{2} \int_{Q}\left(\tilde{u}-u^{*}\right)^{2} d x d t+\frac{1}{2 \tilde{r}} \int_{Q}\left(\tilde{y}-y^{*}\right)^{2 \tilde{r}} d x d t .
\end{gathered}
$$

Combining (3.2), (3.6), (3.8) and (3.9), we infer that $J_{\varepsilon}(\tilde{y}, \tilde{u})=d$. Thus $(\tilde{y}, \tilde{u})$ is a solution of problem $\left(P^{\varepsilon}\right)$. This completes the proof.

LEMMA 3.2. Let $\left(y_{\varepsilon}, u_{\varepsilon}\right)$ be optimal for problem $\left(P^{\varepsilon}\right)$. Then, on a subsequence of $\varepsilon$, denoted in the same way, $y_{\varepsilon} \rightarrow y^{*}$ strongly in $Y$ and $u_{\varepsilon} \rightarrow u^{*}$ strongly in $L^{2}(Q)$.

PROOF. It is clear that

$$
J_{\varepsilon}\left(y_{\varepsilon}, u_{\varepsilon}\right) \leq J_{\varepsilon}\left(y^{*}, u^{*}\right)
$$

By (3.10) and by a standard argument in [2] (see Theorem 2.2, Proposition 2.15 of Chapter 2), we get that

$$
\underset{\varepsilon \rightarrow 0}{\limsup } J_{\varepsilon}\left(y_{\varepsilon}, u_{\varepsilon}\right) \leq g\left(y^{*}\right)+h\left(u^{*}\right)=J\left(y^{*}, u^{*}\right) .
$$

By virtue of (3.1), (3.11) and hypotheses $\left(H_{2}\right)$ and $\left(H_{3}\right),\left\{y_{\varepsilon}\right\}$ is bounded in $L^{2 \tilde{r}}(Q)$, $\left\{u_{\varepsilon}\right\}$ is bounded in $L^{2}(Q)$ and

$$
\int_{Q}\left[\frac{\partial y_{\varepsilon}}{\partial t}+A y_{\varepsilon}+f\left(x, t, y_{\varepsilon}\right)\right]^{2} d x d t \leq 2 C \varepsilon
$$

for some positive constant $C$ independent of $\varepsilon$.

By the subsequence argument as in the proof of Lemma 3.1, we may assume that

$$
\begin{array}{ll}
y_{\varepsilon} \rightarrow \tilde{y} & \text { weakly in } Y \text { and strongly in } L^{2}(Q), \\
u_{\varepsilon} \rightarrow \tilde{u} & \text { weakly in } L^{2}(Q)
\end{array}
$$


and

$$
\frac{\partial y_{\varepsilon}}{\partial t}+A y_{\varepsilon}+f\left(x, t, y_{\varepsilon}\right) \rightarrow \frac{\partial \tilde{y}}{\partial t}+A \tilde{y}+f(x, t, \tilde{y}) \text { weakly in } L^{2}(Q)
$$

for some $\tilde{y} \in Y$ and $\tilde{u} \in L^{2}(Q)$.

It follows from (3.12) and (3.14) that

$$
\begin{aligned}
& \frac{\partial \tilde{y}}{\partial t}+A \tilde{y}+f(x, t, \tilde{y})=0 \text { in } Q, \\
& \tilde{y}=0 \text { on } \Sigma .
\end{aligned}
$$

By (3.1) and (3.11), we also have that $\left[\varepsilon+d_{S}\left(F\left(y_{\varepsilon}\right)\right)\right]^{2} / 2 \varepsilon \leq C$. This implies that $d_{S}\left(F\left(y_{\varepsilon}\right)\right) \rightarrow 0$ as $\varepsilon \rightarrow 0$. By (3.13) and by hypothesis $\left(H_{6}\right)$, we have that $F\left(y_{\varepsilon}\right) \rightarrow F(\tilde{y})$. Hence

$$
F(\tilde{y}) \in S
$$

Thus it follows from (3.15) and (3.16) that

$$
J\left(y^{*}, u^{*}\right) \leq J(\tilde{y}, \tilde{u})
$$

Since $h$ is weakly lower semicontinuous and $g$ is continuous, we have that

$$
\liminf _{\varepsilon \rightarrow 0}\left[g^{\varepsilon}\left(y_{\varepsilon}\right)+h_{\varepsilon}\left(u_{\varepsilon}\right)\right] \geq g(\tilde{y})+h(\tilde{u}) .
$$

By (3.1), (3.17) and (3.18), we obtain that

$$
\liminf _{\varepsilon \rightarrow 0} J_{\varepsilon}\left(y_{\varepsilon}, u_{\varepsilon}\right) \geq J\left(y^{*}, u^{*}\right)
$$

By (3.1), (3.11), (3.13) and (3.19), we infer that

$$
y_{\varepsilon} \rightarrow y^{*} \quad \text { strongly in } L^{2 \pi}(Q) \text { weakly in } Y \text { as } \varepsilon \rightarrow 0
$$

and

$$
u_{\varepsilon} \rightarrow u^{*} \quad \text { strongly in } L^{2}(Q) \text { as } \varepsilon \rightarrow 0
$$

It is clear that

$$
\begin{aligned}
& \left\|f\left(x, t, y_{\varepsilon}\right)-f\left(x, t, y^{*}\right)\right\|_{L^{2}(Q)} \\
& \quad \leq \int_{Q}\left|y_{\varepsilon}-y^{*}\right| \mid \int_{0}^{1} f_{y}^{\prime}\left(x, t, y^{*}+s\left(y_{\varepsilon}-y^{*}\right) d s \mid d x d t .\right.
\end{aligned}
$$


By $\left(H_{4}\right)$ and by Sobolev's imbedding theorem

$$
\| \int_{0}^{1} f_{y}^{\prime}\left(x, t, y^{*}+s\left(y_{\varepsilon}-y^{*}\right) d s \|_{L^{n}(Q)} \leq C,\right.
$$

where $C>0$ is independent of $\varepsilon$. Thus from (3.20), (3.23) and by applying Sobolev's imbedding theorem and Holder's inequality to (3.22), we obtain that

$$
f\left(x, t, y_{\varepsilon}\right) \rightarrow f\left(x, t, y^{*}\right) \text { strongly in } L^{2}(Q) \text { as } \varepsilon \rightarrow 0 .
$$

By (3.12), we have that

$$
\frac{\partial\left(y_{\varepsilon}-y^{*}\right)}{\partial t}+A\left(y_{\varepsilon}-y^{*}\right)+\left[f\left(x, t, y_{\varepsilon}\right)-f\left(x, t, y^{*}\right)\right]+u_{\varepsilon}-u^{*} \rightarrow 0
$$

strongly in $L^{2}(Q)$ as $\varepsilon \rightarrow 0$, which together with (3.21) and (3.24) yields that $y_{\varepsilon} \rightarrow y^{*}$ strongly in $Y$ as $\varepsilon \rightarrow 0$. This completes the proof.

THEOREM 3.3. Let $\left(y^{*}, u^{*}\right)$ be optimal for problem $(P)$. Suppose that $\left(H_{1}\right)-\left(H_{6}\right)$ hold. Then there exist $p \in L^{2}(Q) \cap L^{2 n / n+2}\left(0, T: W_{0}^{1,2 n / n+2}(\Omega)\right)$ with $\left(\lambda_{0}, \xi_{0}\right) \neq 0$ and $\alpha \in \partial g\left(y^{*}\right)$ such that

$$
\begin{aligned}
& \frac{\partial p}{\partial t}-A p-f_{y}^{\prime}\left(x, t, y^{*}\right) p-\left[F^{\prime}\left(y^{*}\right)\right]^{*} \xi_{0}=\lambda_{0} \alpha \quad \text { in } Q, \\
& p \in \lambda_{0} \partial h\left(u^{*}\right), \\
& \left\langle\xi_{0}, \psi-F\left(y^{*}\right)\right\rangle_{X^{*}, X} \leq 0 \quad \forall \psi \in S .
\end{aligned}
$$

Proof. Let $(z, v) \in Y \times L^{2}(Q)$ be arbitrary but fixed. Set $y_{\varepsilon}^{\rho}=y_{\varepsilon}+\rho z$, $u_{\varepsilon}^{\rho}=u_{\varepsilon}+\rho v, \rho>0$. Then

$$
\frac{J_{\varepsilon}\left(y_{\varepsilon}^{\rho}, u_{\varepsilon}^{\rho}\right)-J_{\varepsilon}\left(y_{\varepsilon}, u_{\varepsilon}\right)}{\rho} \geq 0 \text { for all } \rho>0 .
$$

By the hypotheses $\left(\mathrm{H}_{2}\right)$ and $\left(\mathrm{H}_{3}\right)$, we obtain that

$$
\begin{aligned}
& \int_{0}^{T} \frac{g^{\varepsilon}\left(y_{\varepsilon}^{\rho}\right)-g^{\varepsilon}\left(y_{\varepsilon}\right)}{\rho} d t \rightarrow \int_{0}^{T}\left\langle\nabla g^{\varepsilon}\left(y_{\varepsilon}\right), z\right\rangle d t \quad \text { as } \rho \rightarrow 0, \\
& \int_{0}^{T} \frac{h_{\varepsilon}\left(u_{\varepsilon}^{\rho}\right)-h_{\varepsilon}\left(u_{\varepsilon}\right)}{\rho} d t \rightarrow \int_{0}^{T}\left\langle\nabla h_{\varepsilon}\left(u_{\varepsilon}\right), v\right\rangle d t \quad \text { as } \rho \rightarrow 0 .
\end{aligned}
$$

Here $\nabla g^{\varepsilon}$ and $\nabla h_{\varepsilon}$ are the Fréchet derivatives of $g^{\varepsilon}$ and $h_{\varepsilon}$, respectively.

It is easy to check the following:

$$
\frac{1}{2 \rho} \int_{Q}\left[\left(u_{\varepsilon}^{\rho}-u^{*}\right)^{2}-\left(u_{\varepsilon}-u^{*}\right)^{2}\right] d x d t \rightarrow \int_{Q}\left(u_{\varepsilon}-u^{*}\right) v d x d t \quad \text { as } \rho \rightarrow 0
$$


and

$$
\left.\frac{1}{2 \tilde{r} \rho} \int_{Q}\left[y_{\varepsilon}^{\rho}-y^{*}\right)^{2 \tilde{r}}-\left(y_{\varepsilon}-y^{*}\right)^{2 \dot{r}}\right] d x d t \rightarrow \int_{Q}\left(y_{\varepsilon}-y^{*}\right)^{2 \tilde{r}-1} z d x d t \quad \text { as } \rho \rightarrow 0 \text {. }
$$

By (2.1) and (2.2), one can easily show that as $\rho \rightarrow 0$

$$
\begin{aligned}
\frac{1}{2 \varepsilon \rho} \int_{Q}\{[ & {\left[\frac{\partial y_{\varepsilon}^{\rho}}{\partial t}+A y_{\varepsilon}^{\rho}+f\left(x, t, y_{\varepsilon}^{\rho}\right)-u_{\varepsilon}^{\rho}\right]^{2} } \\
& \left.-\left[\frac{\partial y_{\varepsilon}}{\partial t}+A y_{\varepsilon}+f\left(x, t, y_{\varepsilon}\right)-u_{\varepsilon}\right]^{2} d x d t\right\} \\
\rightarrow & \frac{1}{\varepsilon} \int_{Q}\left(\frac{\partial y_{\varepsilon}}{\partial t}+A y_{\varepsilon}+f\left(x, t, y_{\varepsilon}\right)-u_{\varepsilon}\right) \\
& \times\left(\frac{\partial z}{\partial t}+A z+f_{y}^{\prime}\left(x, t, y_{\varepsilon}\right) z-v\right) d x d t .
\end{aligned}
$$

Using the hypothesis $\left(H_{5}\right)$, we obtain that as $\rho \rightarrow 0$

$$
\frac{\left[\varepsilon+d_{S}\left(F\left(y_{\varepsilon}^{\rho}\right)\right)\right]^{2}-\left[\varepsilon+d_{S}\left(F\left(y_{\varepsilon}\right)\right)\right]^{2}}{2 \varepsilon \rho} \rightarrow \frac{\left[\varepsilon+d_{S}\left(F\left(y_{\varepsilon}\right)\right)\right]}{\varepsilon}\left\langle\xi_{\varepsilon}, F^{\prime}\left(y_{\varepsilon}\right) z\right\rangle_{X \cdot, X},
$$

where

$$
\xi_{\varepsilon} \in \partial d_{S}\left(F\left(y_{\varepsilon}\right)\right)= \begin{cases}\nabla d_{S}\left(F\left(y_{\varepsilon}\right)\right) & \text { if } F\left(y_{\varepsilon}\right) \notin S \\ 0 & \text { if } F\left(y_{\varepsilon}\right) \in S\end{cases}
$$

and

$$
\left\|\xi_{\varepsilon}\right\|_{X^{*}}= \begin{cases}1 & \text { if } F\left(y_{\varepsilon}\right) \notin S \\ 0 & \text { if } F\left(y_{\varepsilon}\right) \in S\end{cases}
$$

Let

$$
\lambda_{\varepsilon}=\frac{\varepsilon}{\varepsilon+d_{S}\left(F\left(y_{\varepsilon}\right)\right)} \quad \text { and } \quad \mu_{\varepsilon}=\frac{1}{\varepsilon+d_{S}\left(F\left(y_{\varepsilon}\right)\right)} .
$$

Then by the definition of $J_{\varepsilon}$ and (3.29)-(3.34), it follows from (3.28) (as $\rho \rightarrow 0$ ) that

$$
\begin{aligned}
\lambda_{\varepsilon} \int_{Q} & {\left[\nabla g^{\varepsilon}\left(y_{\varepsilon}\right) z+\nabla h_{\varepsilon}\left(u_{\varepsilon}\right) v\right] d x d t } \\
& +\lambda_{\varepsilon} \int_{Q}\left(u_{\varepsilon}-u^{*}\right) v d x d t+\lambda_{\varepsilon} \int_{Q}\left(y_{\varepsilon}-y^{*}\right)^{2 \tilde{r}-1} z d x d t \\
& +\mu_{\varepsilon} \int_{Q}\left(\frac{\partial y_{\varepsilon}}{\partial t}+A y_{\varepsilon}+f\left(x, t, y_{\varepsilon}\right)-u_{\varepsilon}\right)\left(\frac{\partial z}{\partial t}+A z+f_{y}^{\prime}\left(x, t, y_{\varepsilon}\right) z-v\right) d x d t \\
& +\int_{Q} F^{\prime}\left(y_{\varepsilon}\right)^{*} \xi_{\varepsilon} z d x d t \geq 0
\end{aligned}
$$


for all $(z, v) \in Y \times L^{2}(Q)$.

Let $p_{\varepsilon}=\mu_{\varepsilon}\left[\partial y_{\varepsilon} / \partial t+A y_{\varepsilon}+f\left(x, t, y_{\varepsilon}\right)-u_{\varepsilon}\right]$. It is clear that $p_{\varepsilon} \in L^{2}(Q)$. Letting $z=0$ in (3.35), we obtain that for all $v \in L^{2}(Q)$

$$
\lambda_{\varepsilon}\left[\int_{Q} \nabla h_{\varepsilon}\left(u_{\varepsilon}\right) v d x d t+\int_{Q}\left(u_{\varepsilon}-u^{*}\right) v d x d t\right]-\int_{Q} p_{\varepsilon} v d x d t \geq 0
$$

which implies that

$$
p_{\varepsilon}=\lambda_{\varepsilon}\left[\nabla h_{\varepsilon}\left(u_{\varepsilon}\right)+\left(u_{\varepsilon}-u^{*}\right)\right] \in L^{2}(Q) \text { a.e. in } Q \text {. }
$$

Letting $v=0$ in (3.35), we obtain that

$$
\begin{aligned}
0= & \lambda_{\varepsilon}\left[\int_{Q} \nabla g^{\varepsilon}\left(y_{\varepsilon}\right) z d x d t+\int_{Q}\left(y_{\varepsilon}-y^{*}\right)^{2 \tilde{r}-1} z d x d t\right] \\
& +\int_{Q} F^{\prime}\left(y_{\varepsilon}\right)^{*} \xi_{\varepsilon} z d x d t+\int_{Q} p_{\varepsilon}\left(\frac{\partial z}{\partial t}+A z+f_{y}^{\prime}\left(x, t, y_{\varepsilon}\right) z\right) d x d t
\end{aligned}
$$

for all $z \in Y$. By $\left(H_{4}\right)$, we obtain that $f_{y}^{\prime}\left(x, t, y_{\varepsilon}\right) p_{\varepsilon} \in L^{2 n /(n+2)}(Q)$ and $\left(y_{\varepsilon}-y^{*}\right)^{2 \tilde{r}-1} \in L^{2 n /(n+2)}(Q)$. On the other hand, it is clear that $\lambda_{\varepsilon} \nabla g^{\varepsilon}\left(y_{\varepsilon}\right) \in L^{2}(Q)$ and $F^{\prime}\left(y_{\varepsilon}\right)^{*} \xi_{\varepsilon} \in L^{2}(Q)$. Thus, by (3.37), we infer that $p \in L^{2 n /(n+2)}\left(0, T ; W_{0}^{2 n /(n+2)}(\Omega)\right)$ and satisfies in $Q$

$$
-\frac{\partial p_{\varepsilon}}{\partial t}+f_{y}^{\prime}\left(x, t, y_{\varepsilon}\right) p_{\varepsilon}+\lambda_{\varepsilon} \nabla g^{\varepsilon}\left(y_{\varepsilon}\right)+\lambda_{\varepsilon}\left(y_{\varepsilon}-y^{*}\right)^{2 \tilde{r}-1}+F^{\prime}\left(y_{\varepsilon}\right)^{*} \xi_{\varepsilon}=0
$$

Note that conditions (3.36) and (3.38) can be regarded as necessary conditions for $\left(y_{\varepsilon}, u_{\varepsilon}\right)$ to be optimal for problems $\left(P^{\varepsilon}\right)$.

Next we are going to pass to the limit in (3.36) and (3.38) and derive necessary conditions for $\left(y^{*}, u^{*}\right)$.

First we deal with (3.36). It is obvious from (3.34) that $0<\lambda_{\varepsilon} \leq 1$. Thus we may assume that

$$
\lambda_{\varepsilon} \rightarrow \lambda_{0}, \quad \xi_{\varepsilon} \rightarrow \xi_{0} \quad \text { weakly star in } X^{*} \text { as } \varepsilon \rightarrow 0
$$

By Lemma 3.2, $u_{\varepsilon} \rightarrow u^{*}$ strongly in $L^{2}(Q)$. Then using a standard argument in [2, Chapter 3], we yield that there exists $\beta \in \partial h\left(u^{*}\right)$, such that

$$
\left.\nabla h_{\varepsilon}\left(u_{\varepsilon}\right) \rightarrow \beta \quad \text { weakly in } L^{(} Q\right) \text { as } \varepsilon \rightarrow 0
$$

Now it follows from Lemma 3.2, (3.36), (3.39) and (3.40) that $\left\{p_{\varepsilon}\right\}$ is bounded in $L^{2}(Q)$. So we may assume that there exists a function $p \in L^{2}(Q)$, such that

$$
p_{\varepsilon} \rightarrow p \quad \text { weakly in } L^{2}(Q) \text { as } \varepsilon \rightarrow 0 \text {. }
$$


By (3.39)-(3.41), we may pass to the limit for $\varepsilon \rightarrow 0$ in (3.36) and obtain that $p \in \lambda_{0} \partial h\left(u^{*}\right)$. This gives (3.26).

Now we consider (3.38). By (2.2), (3.41), Lemma 3.2 and Sobolev's imbedding theorem, we have that, for some constant $C$ independent of $\varepsilon$,

$$
\left\|f_{y}^{\prime}\left(x, t, y_{\varepsilon}\right) p_{\varepsilon}\right\|_{L^{2 n /(n+2)(2)}} \leq C \text {. }
$$

By Lemma 3.2 and (3.39) we infer that there exists $\alpha \in \partial g\left(y^{*}\right)$, such that, on a subsequence of $\varepsilon$, denoted in the same way,

$$
\lambda_{\varepsilon} \nabla g^{\varepsilon}\left(y_{\varepsilon}\right) \rightarrow \lambda_{0} \alpha \quad \text { weakly in } L^{2}(Q) \text { as } \varepsilon \rightarrow 0
$$

By Lemma 3.2 and Sobolev's imbedding theorem, we infer that

$$
\lambda_{\varepsilon}\left(y_{\varepsilon}-y^{*}\right)^{2 \tilde{r}-1} \rightarrow 0 \text { strongly in } L^{2 n /(n+2)}(Q) .
$$

On the other hand, by (3.30), Lemma 3.2 and by $\left(H_{5}\right)$, we obtain that

$$
F^{\prime}\left(y_{\varepsilon}\right)^{*} \xi_{\varepsilon} \rightarrow F^{\prime}\left(y^{*}\right)^{*} \xi_{0} \quad \text { weakly in } L^{2}(Q) \text { as } \varepsilon \rightarrow 0 \text {. }
$$

Thus by (3.38) and (3.42)-(3.45), there exists a function

$$
p \in L^{2 n /(n+2)}\left(0, T: W_{0}^{2 n /(n+2)}(Q)\right)
$$

such that, on a subsequence of $\varepsilon$, denoted in the same way,

$$
p_{\varepsilon} \rightarrow p \quad \text { weakly in } L^{2 n /(n+2)}\left(0, T: W_{0}^{2 n /(n+2)}(Q)\right) \text { as } \varepsilon \rightarrow 0
$$

and

$$
p_{\varepsilon}(x) \rightarrow p(x) \quad \text { a.e. in } Q \text { as } \varepsilon \rightarrow 0 \text {. }
$$

By Lemma 3.2 and (3.47), we can easily imply that

$$
f_{y}^{\prime}\left(x, t, y_{\varepsilon}(x)\right) p_{\varepsilon}(x) \rightarrow f_{y}^{\prime}\left(x, t, y^{*}(x)\right) p(x) \quad \text { a.e. in } Q \text {. }
$$

It follows from (3.42) and (3.48) that

$$
f_{y}^{\prime}\left(x, t, y_{\varepsilon}\right) p_{\varepsilon} \rightarrow f_{y}^{\prime}\left(x, t, y^{*}\right) p \quad \text { weakly in } L^{2 n /(n+2)}(Q) \text { as } \varepsilon \rightarrow 0
$$

Now by (3.43)-(3.46) and (3.49), we may pass to the limit in (3.38) and obtain (3.25).

On the other hand since $\xi_{\varepsilon} \in \partial d_{S}\left(F\left(y_{\varepsilon}\right)\right)$, we have that

$$
\left\langle\xi_{\varepsilon}, \psi-F\left(y_{\varepsilon}\right)\right\rangle_{x \cdot, x} \leq 0 \text { for all } \psi \in S
$$


By Lemma 3.2 and by $\left(H_{5}\right)$, we yield that $F\left(y_{\varepsilon}\right) \rightarrow F\left(y^{*}\right)$ strongly in $X$. Thus for all $\psi \in S$

$$
\left\langle\xi_{\varepsilon}, \psi-F\left(y^{*}\right)\right\rangle_{X \cdot, x} \leq\left\langle\xi_{\varepsilon}, F\left(y_{\varepsilon}\right)-F\left(y^{*}\right)\right\rangle_{X \cdot, X} \rightarrow 0 \text { as } \varepsilon \rightarrow 0
$$

By taking the limit for $\varepsilon \rightarrow 0$ in (3.50), we get (3.27).

It remains to show that $\left(\lambda_{0}, \xi_{0}\right) \neq 0$. First we note that by (3.34),

$$
1 \leq \lambda_{\varepsilon}+\left\|\xi_{\varepsilon}\right\|_{X} \leq 2 \text { for all } \varepsilon>0
$$

Now we suppose $\lambda_{0}=0$. It follows from (3.51) that there exist constants $\delta>0$ and $\varepsilon_{1}>0$ such that

$$
\left\|\xi_{\varepsilon}\right\|_{X^{*}} \geq \delta>0 \quad \text { for all } \varepsilon<\varepsilon_{1} .
$$

Using (3.35), (3.36) and (3.50), we obtain that

$$
\begin{aligned}
& \left\langle\xi_{\varepsilon}, F^{\prime}\left(y^{*}\right) z-\psi+F\left(y^{*}\right)\right\rangle_{X^{*}, x} \\
& \quad+\int_{Q} p_{\varepsilon}\left[\frac{\partial z}{\partial t}+A z+f_{y}^{\prime}\left(x, t, y_{\varepsilon}\right) z-v\right] d x d t \geq-\eta_{\varepsilon}(z, v)
\end{aligned}
$$

for all $(z, v) \in L^{2 n /(n+2)}\left(0, T: W_{0}^{2 n /(n+2)}(Q)\right) \times L^{2}(Q)$, where

$$
\begin{aligned}
\eta_{\varepsilon}(z, v)= & \lambda_{\varepsilon}\left\{\int_{Q}\left[\nabla g^{\varepsilon}\left(y_{\varepsilon}\right) z+\nabla h_{\varepsilon}\left(u_{\varepsilon}\right) v\right] d x\right. \\
& \left.+\int_{Q}\left(u_{\varepsilon}-u^{*}\right) v d x d t+\int_{Q}\left(y_{\varepsilon}-y^{*}\right)^{2 \bar{r}-1} z d x d t\right\} \\
& +\left\langle\xi_{\varepsilon},\left[F^{\prime}\left(y_{\varepsilon}\right)-F^{\prime}\left(y^{*}\right)\right] z+F\left(y_{\varepsilon}\right)-\left.F\left(y^{*}\right)\right|_{X^{*}, X}\right.
\end{aligned}
$$

For any $z \in D_{r}$ and every $\varepsilon>0$, by taking $v_{\varepsilon}(z)=\partial z / \partial t+A z+f_{y}^{\prime}\left(x, t, y_{\varepsilon}\right) z$ in (3.53), we deduce that

$$
\left\langle\xi_{\varepsilon}, F^{\prime}\left(y^{*}\right) z-\psi+F\left(y^{*}\right)\right\rangle_{X^{*}, X} \geq-\eta_{\varepsilon}\left(z, v_{\varepsilon}(z)\right)
$$

for all $z \in D_{r}$ and $\varepsilon>0$. By $\left(H_{4}\right)$ and by Lemma 3.2, there exists a positive constant, denoted by $\varepsilon_{1}>0$ again, such that $\left\|f_{y}^{\prime}\left(x, t, y_{\varepsilon}\right)\right\|_{L^{n}(Q)} \leq C$ for all $\varepsilon<\varepsilon_{1}$, where $C$ is a constant independent of $\varepsilon$. Then by Holder's inequality and by Sobolev's imbedding theorem, we infer that

$$
\left\|f_{y}^{\prime}\left(x, t, y_{\varepsilon}\right) z\right\|_{L^{2}(Q)} \leq C r
$$

for all $z \in D_{r}$ and $\varepsilon<\varepsilon_{1}$, where $C$ is independent of $\varepsilon$ and $z$. 
Using the definition of $v_{\varepsilon}$ and $D_{r}$ and (3.56), we check that

$$
\left\|v_{\varepsilon}(z)\right\|_{L^{2}(Q)} \leq C \text { for all } z \in D_{r} \text { and } \varepsilon<\varepsilon_{1} .
$$

By $\left(H_{5}\right)$ and by (3.50), we infer that

$$
\left\langle\xi_{\varepsilon},\left[F^{\prime}\left(y_{\varepsilon}\right)-F^{\prime}\left(y^{*}\right)\right] z\right\rangle_{X \cdot, X} \rightarrow 0, \quad \text { uniformly in } z \in D_{r} .
$$

Now it follows from (3.54), (3.57) and (3.58) that

$$
\eta_{\varepsilon}\left(z, v_{\varepsilon}(z)\right) \rightarrow 0 \quad \text { as } \varepsilon \rightarrow 0 \text {, uniformly in } z \in D_{r} \text {. }
$$

By $\left(H_{6}\right), F^{\prime}\left(y^{*}\right) D_{r}-S+\left\{F\left(y^{*}\right)\right\}$ has finite codimensionality in $X$. Thanks to [4] (see Lemma 3.6 of Chapter 4), we conclude by (3.52), (3.55) and (3.59) that $\xi_{0} \neq 0$. Hence

$$
\left(\lambda_{0}, \xi_{0}\right) \neq 0
$$

Finally, if $F^{\prime}\left(y^{*}\right)^{*}$ is injective and $\lambda_{0}=0$, then by (3.26), we have $p=0$. Thus it follows from (3.25) that $F^{\prime}\left(y^{*}\right)^{*} \xi_{0}=0$ which implies that $\xi_{0}=0$. This contradicts (3.60). So $\lambda_{0} \neq 0$ in the case that $F^{\prime}\left(y^{*}\right)^{*}$ is injective. This completes the proof.

\section{Application}

In this section we apply Theorem 3.3 to a non-well-posed parabolic system.

Let $Q=\Omega \times(0, T), \Omega \subset R^{3}$ be a bounded domain with smooth boundary $\partial \Omega$. We consider the following problem $\left(P^{\prime}\right)$ :

$$
\text { determine } \inf J(y, u)=\inf \int_{0}^{T}[g(t, y(t))+h(u(t))] d t
$$

such that

$$
\begin{aligned}
& \frac{\partial y(x, t)}{\partial t}-\Delta y(x, t)=y^{3}(x, t)+u(x, t) \quad \text { in } Q, \\
& y(x, t)=0 \quad \text { on } \quad \Sigma, \quad y(x, 0)=y_{0}
\end{aligned}
$$

with the state constraint $F(y) \subset S$.

We consider the control set to be $U=\{u: Q \rightarrow R: m \leq u(x, t) \leq M\}$ where $m<M$. Let $f(x, t, y)=-y^{3}$. One can easily check that $f$ satisfies all the conditions in $\left(H_{4}\right)$. For each $u \in L^{2}(Q)$, system (4.1) has, in general, no global solution (see for example [6, Chapter 7]). This is a non-well-posed system. Moreover, we assume that $g, h$ and $F$ satisfy $\left(H_{2}\right),\left(H_{3}\right),\left(H_{5}\right)$ and $\left(H_{6}\right)$. Then the following theorem is immediate from Theorem 3.3. 
THEOREM 4.1. Let $\left(y^{*}, u^{*}\right)$ be optimal for problem $\left(P^{\prime}\right)$. Suppose that $\left(H_{2}\right),\left(H_{3}\right)$, $\left(H_{5}\right)$ and $\left(H_{6}\right)$ hold. Then there exists $p \in L^{2}(Q) \cap L^{6 / 5}\left(0, T: W_{0}^{1,6 / 5}(\Omega)\right)$ with $\left(\lambda_{0}, \xi_{0}\right) \neq 0$ and $\alpha \in \partial g\left(y^{*}\right)$ such that

$$
\begin{aligned}
& \frac{\partial p(x, t)}{\partial t}+\Delta p(x, t)-3 y^{* 2}(x, t) p(x, t)-\left[F^{\prime}\left(y^{*}(x, t)\right)\right]^{*} \xi_{0}=\lambda_{0} \alpha \text { in } Q, \\
& p \in \lambda_{0} \partial h\left(u^{*}\right), \\
& \left\langle\xi_{0}, \psi-F\left(y^{*}\right)\right\rangle_{X^{\cdot}, x} \leq 0 \quad \forall \psi \in S .
\end{aligned}
$$

\section{References}

[1] V. Barbu, Analysis and control of nonlinear infinite dimensional systems (Academic Press, Boston, 1993).

[2] V. Barbu, Optimal control of variational inequalities, Pitman Research Notes in Mathematics 100 (Pitman, London, 1994).

[3] E. Casas, M. Mateos and J. P. Raymond, "Pontryagin's principle for the control of parabolic equations with gradient state constraints", Nonlinear Anal. 46 (2001) 933-956.

[4] X. Li and J. Yong, Optimal control theory for infinite dimensional systems (Birhauser, Boston, 1995).

[5] J. L. Lions, Optimal control of systems governed by partial differential equations (Springer, Berlin, 1971).

[6] J. L. Lions, Some methods in mathematical analysis of systems and their control (Science Press, Beijing, China, Gordon and Breach, New York, 1981).

[7] J. P. Raymond and H. Zidani, "Pontryagin's principle for time-optimal problems", J. Optim. Theory Appl. 101 (1999) 375-402.

[8] G. S. Wang, "Optimal control problems governed by non-well-posed semilinear elliptic equation", Nonlinear Anal. 49 (2002) 315-333.

[9] G. S. Wang, "Pontryagin's maximum principle of optimal control governed by some non-wellposed semilinear parabolic differential equations", Nonlinear Anal. 53 (2003) 601-618.

[10] G. S. Wang and L. Wang, "Maximum principle for optimal control of non-well-posed elliptic differential equations", Nonlinear Anal. 52 (2003) 41-67. 